\title{
Multielectron effects on the orientation dependence and photoelectron angular distribution of multiphoton ionization of $\mathrm{CO}_{2}$ in strong laser fields
}

\author{
Sang-Kil Son (손상길) $)^{1, *}$ and Shih-I Chu (朱時宜) $)^{1,2, \dagger}$ \\ ${ }^{1}$ Department of Chemistry, University of Kansas, Lawrence, Kansas 66045, USA \\ ${ }^{2}$ Center for Quantum Science and Engineering, Department of Physics, National Taiwan University, Taipei 10617, Taiwan
}

(Received 30 April 2009; published 24 July 2009)

\begin{abstract}
We perform an ab initio study of multiphoton ionization (MPI) of carbon dioxide in intense linearly polarized laser pulses with arbitrary molecular orientation by means of a time-dependent density-functional theory (TDDFT) with proper long-range potential. We develop a time-dependent Voronoi-cell finite difference method with highly adaptive molecular grids for accurate solution of the TDDFT equations. Our results demonstrate that the orientation dependence of MPI is determined by multiple orbital contributions and that the electron correlation effects are significant. The maximum peak of MPI is predicted to be at $40^{\circ}$ in good agreement with recent experimental data. Photoelectron angular distribution reveals the delicate relation between the orientation dependence and the molecular orbital symmetry.
\end{abstract}

DOI: 10.1103/PhysRevA.80.011403

The study of multiphoton ionization (MPI) of molecules in intense ultrashort laser fields is a subject of much current interest in strong-field molecular physics. Recent advances of laser technology enable us to align ensembles of molecules periodically by creating a coherent superposition of rotational states [1]. This laser-induced molecular alignment capability has significant impact on strong-field molecular physics leading to impressive applications such as tomographic imaging of molecular orbitals [2], quantum interference [3] and multiple orbital contribution [4] in high harmonic generation (HHG), and time-resolved photoelectron angular distribution (PAD) [5]. It also has been possible to measure ionization yields [6,7], HHG [8], and PAD [9] as a function of the angle between the polarization of linearly polarized laser field and the orientation of aligned linear molecules. In particular, the field-molecule orientation dependence of MPI has attracted much attention because not only the ionization mechanism plays a fundamental role in strong-field processes but also the molecular alignment may offer additional degree of freedom to control molecular processes.

For strong-field MPI, there have been a number of theoretical studies of diatomic molecules such as $\mathrm{N}_{2}$ and $\mathrm{O}_{2}$ in agreement with the experimental measurements [10-14]. However, for other diatomic molecules such as $F_{2}$, experimental data [15] cannot be explained by approximate models which consider only the highest occupied molecular orbital (HOMO) contribution such as the molecular AmmosovDelone-Krainov (MO-ADK) [13] or Keldysh-Faisal-Reiss [14] models. The electron correlation and inner-molecular orbital contributions are found to be significant for $\mathrm{F}_{2}$ MPI processes [10-12]. For strong-field MPI of triatomic molecules such as $\mathrm{CO}_{2}$, so far there has been no ab initio study available taking into account the detailed electronic structure and multielectron response. Also, significant discrepancy exists between recent experimental data and approximate models regarding the orientation dependence of MPI [6]. Thus it is desirable to develop an $a b$ initio method for polyatomic

\footnotetext{
*sangkil@ku.edu

†sichu@ku.edu
}

PACS number(s): 33.80.Rv, 02.70.-c, 31.15.ee, 42.50.Hz

molecular systems to explore detailed strong-field electronic dynamics including multielectron responses. Although $a b$ initio calculations for molecules in strong fields with arbitrary orientation require extensive computational resources, it has become feasible for diatomic molecules by means of the two-center time-dependent generalized pseudospectral method recently developed $[10,16]$. In this Rapid Communication, we present a grid-based method designed for polyatomic molecular systems and present an ab initio allelectron time-dependent density-functional theory (TDDFT) calculation in order to investigate the orientation dependence and PAD of MPI of the three-center $\mathrm{CO}_{2}$ molecule.

The electronic structure of $\mathrm{CO}_{2}$ is solved by the allelectron density-functional theory (DFT), with proper longrange potential. We generalize the Voronoi-cell finite difference (VFD) method [17] to solve the Schrödinger/KohnSham equations for polyatomic molecules. In contrast to the ordinary finite difference method with regular uniform grids, the VFD method can accommodate any type of grid distributions, so-called unstructured grids, with the help of geometrical flexibility of the Voronoi diagram. To attack multicenter Coulombic singularity in all-electron calculations of polyatomic molecules, we use highly adaptive molecular grids. Molecular grids are intuitively composed of spherical atomic grids centered at each nuclear position regardless of the coordinate system and molecular symmetry. Figure 1 exhibits a two-dimensional (2D) sketch of molecular grids suit-

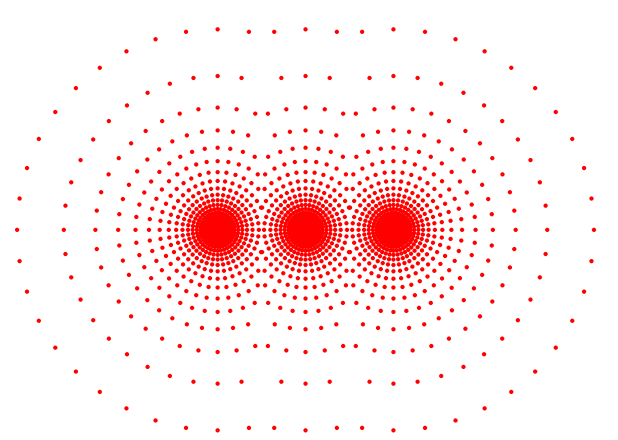

FIG. 1. (Color online) 2D sketch of molecular grids for $\mathrm{CO}_{2}$ used in the Voronoi-cell finite difference method. 
TABLE I. Comparison of absolute orbital binding energies of $\mathrm{CO}_{2}$ computed with $\mathrm{LB} \alpha$ and LDA, and experimental vertical ionization potentials (in $\mathrm{eV}$ ).

\begin{tabular}{lccr}
\hline \hline Orbital & Experiment & LB $\alpha$ & LDA \\
\hline $1 \pi_{g}$ & $13.8^{\mathrm{a}}$ & 13.9 & 8.9 \\
$1 \pi_{u}$ & $17.6^{\mathrm{a}}$ & 17.5 & 12.6 \\
$3 \sigma_{u}$ & $18.1^{\mathrm{a}}$ & 17.2 & 12.3 \\
$4 \sigma_{g}$ & $19.4^{\mathrm{a}}$ & 18.5 & 13.5 \\
$2 \sigma_{u}$ & $36.9^{\mathrm{b}}$ & 32.4 & 27.7 \\
$3 \sigma_{g}$ & $38.0^{\mathrm{b}}$ & 33.5 & 28.8 \\
$2 \sigma_{g}$ & $297.5^{\mathrm{c}}$ & 293.2 & 273.8 \\
$1 \sigma_{u}$ & $540.8^{\mathrm{c}}$ & 540.2 & 516.7 \\
$1 \sigma_{g}$ & $540.8^{\mathrm{c}}$ & 540.2 & 516.8 \\
\hline \hline
\end{tabular}

${ }^{\mathrm{a}}$ Reference [20].

${ }^{\mathrm{b}}$ Reference [21].

${ }^{\mathrm{c}}$ Reference [22].

able for $\mathrm{CO}_{2}$ assuring more grids around nuclei. Note that all calculations are performed in 3D real spaces. In the VFD method, the symmetric discrete Laplacian matrix is simply expressed by distance, area, and volume related to Voronoi cells. Moreover, the potential matrix is diagonal given by a value at each grid, which remarkably simplifies timepropagation procedures. Detailed computational procedures will be reported elsewhere [18].

For an exchange-correlation (xc) functional of DFT calculations, we use the modified Leeuwen-Baerends ( $\mathrm{LB} \alpha$ ) potential [19] that produces the proper long-range Coulombic $(-1 / r)$ behavior. We use $\alpha=1.16$ and $\beta=0.01$ for the $\operatorname{LB} \alpha$ parameters. The ground-state electronic configuration of $\mathrm{CO}_{2}$ is [core] $\left(3 \sigma_{g}\right)^{2}\left(2 \sigma_{u}\right)^{2}\left(4 \sigma_{g}\right)^{2}\left(3 \sigma_{u}\right)^{2}\left(1 \pi_{u}\right)^{4}\left(1 \pi_{g}\right)^{4}$ where [core] indicates $1 s$ orbitals of $\mathrm{C}$ and two O's. Table I compares experimental vertical ionization potentials [20-22] of $\mathrm{CO}_{2}$ and absolute values of orbital binding energies computed with $\mathrm{LB} \alpha$ and local-density approximation (LDA). Molecular grids are constructed by a combination of spherical atomic grids (80 radial and 121 angular grids) covering large distances $\left(r_{\max } \sim 20 \AA\right)$. The bond length of $\mathrm{C}-\mathrm{O}$ is fixed at $1.162 \AA[23]$. Note that the calculated $\operatorname{LB} \alpha$ orbital binding energies are in good agreement with the experimental data, particularly those for HOMO $\left(1 \pi_{g}\right)$ and HOMO-1 $\left(1 \pi_{u}\right)$, well within $0.1 \mathrm{eV}$. In contrast, the LDA values are too weakly bound for all valence orbitals since the LDA xc functional contains self-interaction error and does not have the correct long-range $(-1 / r)$ potential [12]. The $\mathrm{LB} \alpha$ xc potential has the proper long-range potential and much better orbital energies. The small discrepancy of the $\operatorname{LB} \alpha$ inner orbital energies does not affect the present study since the HOMO dominates the process in this case.

We further solve a set of time-dependent Kohn-Sham equations for $N$-electron systems in the TDDFT framework,

$$
i \frac{\partial}{\partial t} \psi_{i \sigma}(\mathbf{r}, t)=\left[-\frac{1}{2} \nabla^{2}+v_{\mathrm{eff}, \sigma}(\mathbf{r}, t)\right] \psi_{i \sigma}(\mathbf{r}, t),
$$

where $i$ and $\sigma$ are the orbital and the spin index, respectively. The effective potential is expressed by $v_{\mathrm{eff}, \sigma}(\mathbf{r}, t)=v_{\mathrm{ne}}(\mathbf{r})$

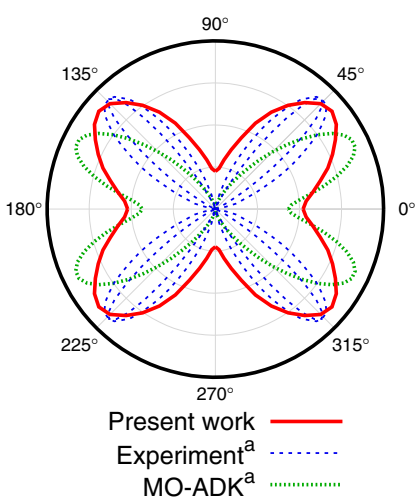

(a) $820 \mathrm{~nm}, 1.1 \times 10^{14} \mathrm{~W} / \mathrm{cm}^{2}$

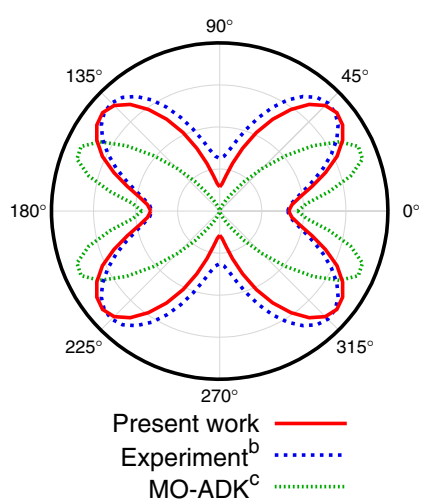

(b) $800 \mathrm{~nm}, 5 \times 10^{13} \mathrm{~W} / \mathrm{cm}^{2}$
FIG. 2. (Color online) Orientation dependence of total ionization probability of $\mathrm{CO}_{2}$. ${ }^{\mathrm{a}}$ Ref. [6]; ${ }^{\mathrm{b}}$ Ref. [7]; and ${ }^{\mathrm{c}}$ Ref. [26].

$+v_{\mathrm{h}}(\mathbf{r}, t)+v_{\mathrm{xc}, \sigma}(\mathbf{r}, t)+\mathbf{F}(t) \cdot \mathbf{r} \quad$ where $\quad v_{\mathrm{ne}}$ is the nucleuselectron Coulomb interaction, $v_{\mathrm{h}}$ is the Hartree potential, and $v_{\mathrm{xc}, \sigma}$ is the exchange-correlation potential. The last term is the interaction of an electron with a linearly polarized external laser field. Without loss of generality, we assume that the molecular axis coincides with the $z$ axis and the field polarization lies in the $x z$ plane, then we have $\mathbf{F}(t) \cdot \mathbf{r}$ $=F(t)(x \sin \Theta+z \cos \Theta)$ where $\Theta$ is the orientation angle between the molecular axis and the polarization axis of the laser field. For the sine-squared pulse envelope, we use $F(t)=F_{0} \sin ^{2}(\pi t / T) \sin \omega t$, where $F_{0}$ is the peak field amplitude, $\omega$ is the carrier frequency, and $T$ is the pulse duration.

For numerical solutions of Eq. (1), we develop a timedependent Voronoi-cell finite difference (TDVFD) method as an extension of the second-order split-operator technique in the energy representation [24,25],

$$
\psi_{i \sigma}(t+\Delta t)=e^{-i \hat{V}(t) \Delta t / 2} e^{-i \hat{H}_{0} \Delta t} e^{-i \hat{V}(t) \Delta t / 2} \psi_{i \sigma}(t)+O\left(\Delta t^{3}\right),
$$

where $\hat{H}_{0}=-\frac{1}{2} \nabla^{2}+v_{\text {eff, } \sigma}(\mathbf{r}, 0)$ and $\hat{V}(t)=\mathbf{F}(t) \cdot \mathbf{r}+\left[v_{\mathrm{xc}, \sigma}(\mathbf{r}, t)\right.$ $\left.-v_{\mathrm{xc}, \sigma}(\mathbf{r}, 0)\right]+\left[v_{\mathrm{h}}(\mathbf{r}, t)-v_{\mathrm{h}}(\mathbf{r}, 0)\right]$. The time-independent Hamiltonian $\hat{H}_{0}$ is solved by the VFD method and the timedependent potential $\hat{V}(t)$ is given by a diagonal matrix evaluated at each grid. Here $\Delta t$ is about 0.1 a.u. in our calculations. All orbitals except core orbitals participate in time propagation by Eq. (2) including multielectron responses in multiple orbital dynamics.

From the TDDFT solutions, one can compute the timedependent ionization probability of each spin-orbital by $P_{i \sigma}(t)=1-N_{i \sigma}(t)$ where $N_{i \sigma}(t)=\left\langle\psi_{i \sigma}(\mathbf{r}, t) \mid \psi_{i \sigma}(\mathbf{r}, t)\right\rangle$ is the population or survival probability of the spin-orbital that decreases as a function of $t$ due to the absorber to filter out the ionized wave packet [24]. The total ionization probability $P$ after one pulse $(t=T)$ can be calculated by

$$
P=1-\prod_{i \sigma}\left[1-P_{i \sigma}(T)\right] .
$$

Figure 2 shows the orientation dependence of the total ionization probability calculated by Eq. (3) as a function of $\Theta$. We used the 20-optical-cycle $\sin ^{2}$-envelope laser pulses with two different sets of the wavelength and the peak intensity: 

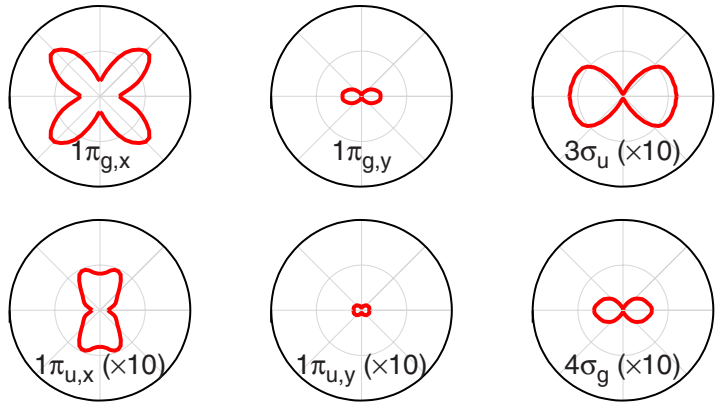

FIG. 3. (Color online) Orientation dependence of individual ionization probability of multiple orbitals with $820 \mathrm{~nm}$ and 1.1 $\times 10^{14} \mathrm{~W} / \mathrm{cm}^{2}$.

(a) $820 \mathrm{~nm}$ and $1.1 \times 10^{14} \mathrm{~W} / \mathrm{cm}^{2}$, and (b) $800 \mathrm{~nm}$ and 5 $\times 10^{13} \mathrm{~W} / \mathrm{cm}^{2}$. For comparison, Fig. 2 includes experimental measurements of Pavičić et al. [6] and Thomann et al. [7] and MO-ADK results $[6,26]$ with corresponding wavelength and peak intensity. All data sets are normalized to their maximum value. In Fig. 2(a), two dashed lines of experiment are due to uncertainty of the measured alignment distribution [6]. The total ionization probability computed from TDDFT manifests the center-fat propeller shape with the maximum peak at $40^{\circ}$, which agrees well with both experimental data of $45^{\circ}$ peak $[6,7]$. As for the broadness of the central pattern, our results are in good agreement with the data of Thomann et al. [7] but different from the data of Pavičić et al. [6], the latter showing a narrower pattern. The cause of this discrepancy is unknown but might be related to the experimental uncertainty in the molecular alignment processes. Note that in theory orientation dependence tends to become less anisotropic at higher intensity [10]. On the other side, the MOADK model predicts the $25^{\circ}$-peak butterfly shape for both cases $[6,26]$. We note that a recent study using the strongfield approximation model predicts that the peak maximum occurs at $37^{\circ}$ [27].

We now examine contributions of individual orbitals on the total ionization probability. The total ionization probability in Eq. (3) can be approximately reduced to the summation of those individual probabilities in the limit of small $P_{i \sigma}(T)$, i.e., $P \approx \sum_{i \sigma} P_{i \sigma}(T)$. Figure 3 contains individual ionization probabilities $P_{i \sigma}(T)$ of multiple orbitals with $820 \mathrm{~nm}$ and $1.1 \times 10^{14} \mathrm{~W} / \mathrm{cm}^{2}$. HOMO $\left(1 \pi_{g}\right)$ is dominant in the total ionization and others $\left(1 \pi_{u}, 3 \sigma_{u}\right.$, and $\left.4 \sigma_{g}\right)$ are scaled by ten times. Contributions of $2 \sigma_{u}$ and $3 \sigma_{g}$ are negligible. In fact, the unperturbed $\pi$ orbitals are degenerate: one lies on the $x z$ plane $\left(1 \pi_{g, x}\right.$ and $\left.1 \pi_{u, x}\right)$ and the other lies on the $y z$ plane $\left(1 \pi_{g, y}\right.$ and $\left.1 \pi_{u, y}\right)$. As the field whose polarization vector varies in the $x z$ plane is applied to the molecule, $1 \pi_{g, x}$ provides the most dominant contribution to the orientation dependence of the total ionization probability, which has the $45^{\circ}$-peak maximum. $1 \pi_{g, y}$ shows a dumbbell shape with the same probability as $1 \pi_{g, x}$ at $\Theta=0^{\circ}$. Thus, the center-fat propeller shape of the total ionization probability in Fig. 2 is mostly reflected by contributions of two HOMOs $\left(1 \pi_{g, x}\right.$ and $\left.1 \pi_{g, y}\right)$.

This prediction is particularly different from the MOADK butterfly pattern based on the structure and symmetry of the ground state of HOMO [26,28]. In MO-ADK, it is understood that the peak angle for $\mathrm{CO}_{2}$ becomes lower than for $\mathrm{O}_{2}$, despite their same HOMO symmetry, because the internuclear distance between two O's in $\mathrm{CO}_{2}$ $(d=2.324 \AA)$ is longer than in $\mathrm{O}_{2}(d=1.210 \AA)$ [28]. In contrast, our TDDFT results incorporating multielectron effects reveal that the orientation dependence of ionization probability of $\mathrm{HOMO}$ peaks at $45^{\circ}$ similar to the predicted pattern for $\mathrm{O}_{2}$ and $\mathrm{F}_{2}$ that have the same HOMO symmetry [10]. Our all-electron $a b$ initio study demonstrates the importance of including the electron correlation and all the valence orbitals in the study of strong-field ionization mechanism even when HOMO is dominant in the ionization process.

To explore the orientation dependence of MPI and its relation to molecular orbital symmetry further, we examine PAD to visualize where the electron is ionized in the strong field as the field-molecule orientation changes. We extend the TDDFT solutions to calculate angular differential ionization probability (ADIP) for the complete PAD picture. The ADIP of each spin-orbital can be expressed as

$$
\frac{\partial P_{i \sigma}}{\partial \Omega}=\int_{0}^{T} r_{0}^{2} \operatorname{Im}\left[\psi_{i \sigma}^{*}(\mathbf{r}, t) \frac{\partial}{\partial r} \psi_{i \sigma}(\mathbf{r}, t)\right]_{r=r_{0}} d t,
$$

where $r_{0}$ is chosen to be less than the absorber position. Then the total ADIP is calculated in the same way as in Eq. (3), $\partial P / \partial \Omega=1-\Pi_{i \sigma}\left(1-\partial P_{i \sigma} / \partial \Omega\right)$.

Figure 4 displays PAD at several field-molecule orientation angles: (a) $\Theta=0^{\circ}$, (b) $\Theta=40^{\circ}$, and (c) $\Theta=90^{\circ}$. Laser parameters are the same ones used in Fig. 2(a) and 256 angular grids are used for each atom. The orientation dependence of MPI has the maximum peak at $\Theta=40^{\circ}$, so Fig. 4(a) is scaled by three times and Fig. 4(c) by five times for clarity. The top and middle panels represent contour maps of $\partial P / \partial \Omega$ as a function of $\Omega$ on the unit sphere in $3 \mathrm{D}$. In Fig. 4, the PAD contour maps show characteristic features of the perturbed HOMO symmetry, because HOMO dominantly contributes to the total ionization. At $\Theta=0^{\circ}$ [Fig. 4(a)], PAD forms a donut shape with a nodal point at the center, which is equally from two HOMOs $\left(1 \pi_{g, x}\right.$ and $\left.1 \pi_{g, y}\right)$, indicating the retained axial symmetry and degeneracy. At $\Theta=90^{\circ}$ [Fig. 4(c)], PAD forms a dumbbell shape with a nodal plane, which is mostly from one HOMO $\left(1 \pi_{g, x}\right)$. Because of the nodal spot and plane at the field direction, the total ionization is suppressed at these orientation angles. On the other hand, PAD at $\Theta=40^{\circ}$ [Fig. 4(b)] shows a peak spot coincided with the field axis, thus enhancing the total ionization in this direction. It strongly supports the maximum peak of the orientation dependence at this angle.

To closely observe PAD with respect to the field direction, the polar angle $\theta$ and the azimuth angle $\phi$ are defined with respect to the field polarization axis, and then the polar plot as a function of $\theta$ is obtained by $\partial P / \partial \theta=\int_{0}^{2 \pi}(\partial P / \partial \Omega) d \phi$. The bottom panels in Fig. 4 represent $\partial P / \partial \theta$ at three orientations. These plots are comparable with conventional PAD 2D polar plots $[9,29]$. Our results indicate the significant advantage of using the 3D PAD visualization, since it provides dynamical information not realizable by the conventional 2D polar plot. For example, while the patterns of the bottom panels in Figs. 4(a) and 4(c) look alike, the corresponding 3D patterns are completely different. 

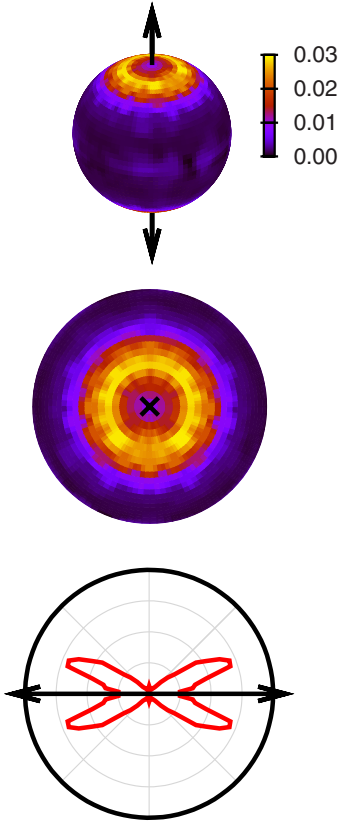

(a) $\Theta=0^{\circ}(\times 3)$
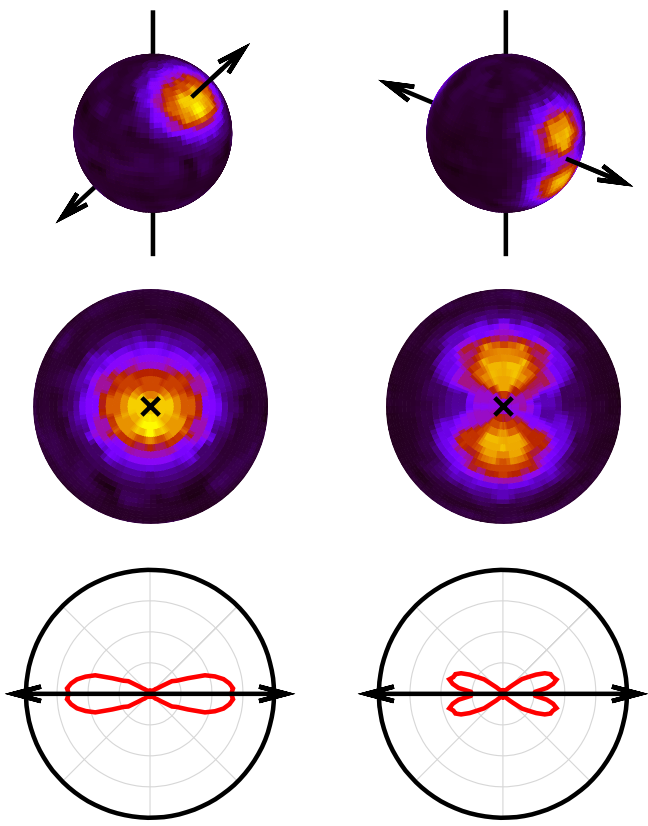

(b) $\Theta=40^{\circ}$

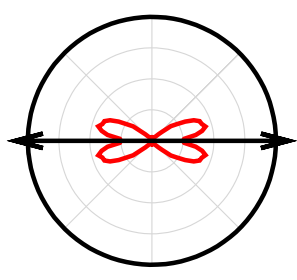

(c) $\Theta=90^{\circ}(\times 5)$

FIG. 4. (Color online) Photoelectron angular distribution at several orientation angles. The top panels display contour maps of $\partial P / \partial \Omega$ on the unit sphere with a vertical line of the molecular axis and an arrow line of the field polarization axis. The middle panels show the same spherical contour maps in different views that the field axis is perpendicular to the paper. The bottom panels represent polar plots of $\partial P / \partial \theta$ with a horizontal arrow line of the field axis.

In summary, we performed TDDFT calculations for $\mathrm{CO}_{2}$ by means of the TDVFD method with highly adaptive molecular grids. Calculated orientation-dependent plot of $\mathrm{CO}_{2}$ MPI shows the center-fat propeller shape with $40^{\circ}$ maximum, which is mainly contributed by two perturbed HOMOs. It agrees well with the recent experiments except the narrowness of the one experimental case. The PAD with various orientations illustrates characteristics of the HOMO symmetry including the nodal shapes and the peak spot at the field direction, explaining suppression and enhancement of MPI at corresponding orientation. It supports the relation be- tween the orientation dependence of MPI and the orbital symmetry.

This work was partially supported by U.S. Department of Energy and U.S. National Science Foundation. We also would like to acknowledge the partial support of National Science Council of Taiwan (Grant No. 97-2112-M-002-003MY3) and National Taiwan University (Grant No. 97R0066). S.K.S. would like to thank Isabell Thomann, Dr. Wen Li, and Dr. Domagoj Pavičić for useful discussions and allowance to use their experimental data.
[1] F. Rosca-Pruna and M. J. J. Vrakking, Phys. Rev. Lett. 87, 153902 (2001); H. Stapelfeldt and T. Seideman, Rev. Mod. Phys. 75, 543 (2003).

[2] J. Itatani et al., Nature (London) 432, 867 (2004).

[3] T. Kanai et al., Nature (London) 435, 470 (2005).

[4] B. K. McFarland et al., Science 322, 1232 (2008).

[5] C. Z. Bisgaard et al., Science 323, 1464 (2009).

[6] D. Pavičić, K. F. Lee, D. M. Rayner, P. B. Corkum, and D. M. Villeneuve, Phys. Rev. Lett. 98, 243001 (2007).

[7] I. Thomann et al., J. Phys. Chem. A 112, 9382 (2008).

[8] R. de Nalda et al., Phys. Rev. A 69, 031804(R) (2004).

[9] V. Kumarappan et al., Phys. Rev. Lett. 100, 093006 (2008).

[10] D. A. Telnov and S. I. Chu, Phys. Rev. A 79, 041401(R) (2009).

[11] X. Chu and S. I. Chu, Phys. Rev. A 70, 061402(R) (2004).

[12] S. I. Chu, J. Chem. Phys. 123, 062207 (2005).

[13] X. M. Tong, Z. X. Zhao, and C. D. Lin, Phys. Rev. A 66, 033402 (2002).

[14] J. Muth-Böhm, A. Becker, and F. H. M. Faisal, Phys. Rev. Lett. 85, 2280 (2000).

[15] M. J. DeWitt, E. Wells, and R. R. Jones, Phys. Rev. Lett. 87, 153001 (2001); E. Wells, M. J. DeWitt, and R. R. Jones, Phys.
Rev. A 66, 013409 (2002).

[16] D. A. Telnov and S. I. Chu, Phys. Rev. A 76, 043412 (2007).

[17] N. Sukumar, Int. J. Numer. Methods Eng. 57, 1 (2003); N. Sukumar and J. E. Bolander, CMES Comput. Model. Eng. Sci. 4, 691 (2003).

[18] S.-K. Son (unpublished).

[19] P. R. T. Schipper et al., J. Chem. Phys. 112, 1344 (2000).

[20] D. W. Turner et al., Molecular Photoelectron Spectroscopy (Wiley-Interscience, London, 1970).

[21] H.-J. Freund et al., Chem. Phys. Lett. 123, 463 (1986).

[22] K. Siegbahn et al., ESCA Applied to Free Molecules (NorthHolland Publishing Co., Amsterdam, 1969).

[23] G. Herzberg, Molecular Spectra and Molecular Structure, III. Electronic Spectra and Electronic Structure of Polyatomic Molecules (Van Nostrand, New York, 1966).

[24] X. M. Tong and S. I. Chu, Chem. Phys. 217, 119 (1997).

[25] X. Chu and S. I. Chu, Phys. Rev. A 63, 023411 (2001).

[26] A.-T. Le et al., J. Mod. Opt. 54, 967 (2007).

[27] V.-H. Le et al., J. Phys. B 41, 085603 (2008).

[28] A. S. Alnaser et al., Phys. Rev. A 71, 031403(R) (2005).

[29] A. Jaroń-Becker et al., J. Phys. B 36, L375 (2003). 